\title{
Lattice damage in 9-MeV-carbon irradiated diamond and its recovery after annealing
}

\author{
F. Agulló-Rueda ${ }^{\mathrm{a}, *}$, N. Gordillo ${ }^{\mathrm{b}, \mathrm{c}}$, M. D. Ynsa ${ }^{\mathrm{b}, \mathrm{c}}$, A. Maira ${ }^{\mathrm{b}}$, J. Cañas ${ }^{\mathrm{b}}$, M. A. Ramos ${ }^{\mathrm{b}, \mathrm{d}}$ \\ ${ }^{a}$ Instituto de Ciencia de Materiales de Madrid (ICMM-CSIC), 28049 Madrid, Spain \\ ${ }^{b}$ Centro de Micro-Análisis de Materiales, Universidad Autónoma de Madrid, 28049 Madrid, Spain \\ ${ }^{c}$ Departamento de Física Aplicada, Universidad Autónoma de Madrid, 28049 Madrid, Spain \\ ${ }^{d}$ Departamento de Física de la Materia Condensada, Condensed Matter Physics Center (IFIMAC) and Instituto Nicolás Cabrera, Universidad Autónoma de \\ Madrid, 28049 Madrid, Spain
}

\begin{abstract}
We have studied the radiation damage in diamond as a function of layer depth upon self-ion implantation with 9-MeV carbon ions and its recovery after annealing at $1000^{\circ} \mathrm{C}$. Raman and photoluminescence spectra show substantial damage of the lattice, namely, amorphization, neutral vacancies, and interstitial defects. Damage is maximum in the stopping layer at a depth of $4 \mu \mathrm{m}$. After annealing there is some recovery of the lattice, but the residual damage increases with fluence, up to about $2 \times 10^{16}$ ions $/ \mathrm{cm}^{2}$. At this fluence the stopping layer becomes highly disordered and does not heal with annealing. Surprisingly, for higher fluence values, of about $5 \times 10^{16}$ ions $/ \mathrm{cm}^{2}$, there is almost no residual damage. After full amorphization is reached, the layers appear to recrystallize by solid phase epitaxy (SPE), using the pristine diamond layers underneath as a template. These results prove that graphitization of diamond after annealing can be avoided in deeply buried layers, implanted at fluences much higher than expected. High fluences, in fact, can lead to high quality diamond layers. If SPE can be confirmed, it would have a great interest for diamond device applications, as it allows for higher doping levels.
\end{abstract}

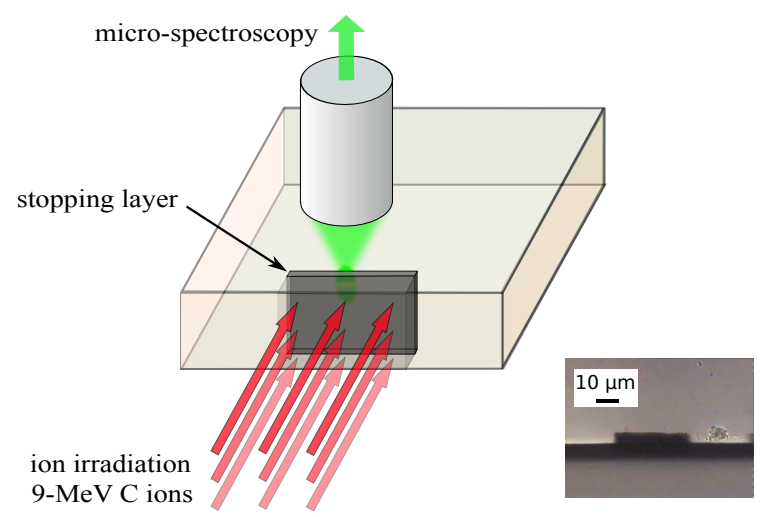

Figure 1: Scheme of the experimental procedure. A diamond crystal was implanted with 9-MeV ${ }^{12} \mathrm{C}^{3+}$ ions at different fluences and then annealed at $1000^{\circ} \mathrm{C}$ for $1 \mathrm{~h}$. Optical microspectroscopy measurements were performed both after implantation and after annealing. The inset shows an actual transmission light micrograph of an implanted stripe as seen from the top sample surface (fluence $130 \times 10^{14}$ ions $/ \mathrm{cm}^{3}$ ).

\section{Introduction}

Diamond is a clear case of a material with outstanding current and future technological applications due to its extreme physical properties, namely being the hardest natural material, with very high Debye temperature and thermal conductivity,

\footnotetext{
Published in Carbon, DOI:10.1016/j.carbon.2017.07.076

${ }^{*}$ Corresponding author

Email address: far@icmm.csic.es (F. Agulló-Rueda)
}

huge electrical resistivity, wide optical transparency window, etc. These potential applications have been limited in the past century because diamond was a very scarce and expensive material. The recent development of techniques for preparing synthetic diamond crystals of high purity and quality has paved the way to exploit these extraordinary properties in many new and exciting applications [1-3].

As a pure material, diamond is an excellent insulator strictly speaking, a very wide bandgap semiconductor $(5.5 \mathrm{eV})$. However its electrical and optical properties can be significantly modified introducing charge carriers by chemical doping [46] or by ion implantation [7-11]. Boron-doped diamond, for example, becomes a $p$-type semiconductor, that could be very useful in microelectronic devices. Further, more heavily borondoped diamond (with boron concentrations of $\approx 4 \times 10^{21} \mathrm{~cm}^{-3}$ ), synthesized by high-pressure/high-temperature techniques [5] or by chemical vapor deposition (CVD) [6], has been found to exhibit superconductivity for temperatures below a few K.

Nevertheless, doping diamond by thermal diffusion or during crystal growth is difficult because of the short and strong carbon-carbon bonds. An appealing alternative is to dope it through ion implantation, which also enables selective doping in a controlled manner when employing focused ion beam irradiation. Its main disadvantage is that after irradiation the crystal lattice is often heavily damaged. In other materials this is easily solved by high-temperature annealing. Under certain conditions the layers amorphized by irradiation can recrystallize by solid phase epitaxy (SPE) in which the amorphous-crystalline interface, originally at the end of range of the ions, moves towards the surface [12]. SPE can produce layers of high quality 
that accept doping levels above those obtained under thermal equilibrium and is well studied in silicon $[13,14]$. The annealing of defects in diamond, however, is a complex issue, since broken carbon bonds can rearrange in different hybridization forms: $s p^{3}$ bonding (diamond), $s p^{2}$ bonding (graphite), or $s p$ bonding (polymer). Also, diamond is a metastable allotrope of carbon, graphite being the stable phase of carbon at atmospheric conditions. As a consequence, above some damage threshold irradiated diamond amorphizes $[15,16]$ and subsequent annealing at temperatures $\approx 1000-1500 \mathrm{~K}$ can lead to crystallization into the more stable phase, i.e. graphite.

Although the damage induced in the diamond lattice by ion implantation is often a drawback to be fixed if possible, in other cases it has been employed as a useful tool. Since graphite is an electrical conductor in contrast to diamond, the abovementioned graphitization of heavily-irradiated diamond after annealing can be used to fabricate conductive micro-channels, microwires or graphitic buried electrodes in the diamond matrix. This kind of three-dimensional micrometric structures, which are the basis for potential electronic and optical devices, have already been manufactured by using microbeams of highenergy (MeV) ions, mainly protons [17] and helium ions [1820], but also with carbon ions [21, 22].

Below the damage threshold, the diamond lattice persists, though containing a number of point defects that can be annealed back to diamond structure, at least partially. By irradiating with different ions in the energy range of tens of $\mathrm{keV}$, several authors [23-25] reported a critical damage of $1 \times 10^{22}$ vacancies $/ \mathrm{cm}^{3}$, above which irradiated diamond graphitized after high-temperature annealing, whereas below it the diamond crystalline lattice was essentially restored. Nonetheless, other studies [18, 26-28] showed that a much higher damage threshold was found when MeV ions were employed, presumably due to an internal pressure effect in those deeply-buried (typically, a few $\mu \mathrm{m}$ ) implanted and damaged layers. This effect would arise from the unusually large differences between the mass densities of the metastable (diamond, $3.52 \mathrm{~g} / \mathrm{cm}^{3}$ ) and stable (graphite, $2.25 \mathrm{~g} / \mathrm{cm}^{3}$ ) phases, that will constrain the strongly damaged diamond structure from relaxing into the graphite one.

The objective of the present work is to exhaustively study the damage caused in diamond crystals by the irradiation of highenergy $(\mathrm{MeV})$ ions and its recovery after thermal annealing, as a function of both the implanted ion dose and layer depth. Indeed, when no particular doping is pursued, an obvious choice is to irradiate diamond with carbon ions, both to produce and to study damage created without introducing a foreign element [21, 22, 29-31]. With such an aim, we have irradiated highpurity diamond single crystals with $9 \mathrm{MeV} \mathrm{C}^{3+}$ microbeams of a few $\mu \mathrm{m}^{2}$, creating micrometric stripes of different ion fluences. Raman spectroscopy has been extensively used to monitor the damage and the transformation of the diamond lattice after ion irradiation [24, 25, 27, 29, 32-37]. Combined Raman and photoluminescence spectroscopy are very sensitive to the residual damage, as shown in our previous paper [37]. In that paper we irradiated diamond with $9 \mathrm{MeV}$ boron ions. In the present paper we have instead implanted carbon ions. We reach much higher fluences, allowing us to get a full amorphization of the end-of-range layer. This resulted in much better recrystallization after annealing, that we believe is due to solid phase epitaxy. Also, in the previous paper we studied only the near-surface layers because the Raman spectra were measured from the same face as the one used for irradiation. In this paper we have measured the Raman spectra along a cross section, allowing us to obtain spectra as a function of layer depth, with a spatial resolution better than $1 \mu \mathrm{m}$. Employing these techniques, in this paper we study the lattice damage in diamond along the ion-irradiation track at $\mu \mathrm{m}$ scale for fluences below and above the amorphization threshold and their behavior after high-temperature annealing, which will produce either vacancy healing in diamond or graphitization. This kind of study of damage in diamond produced when using MeV self-ion microbeam in an all-carbon system had not been done so far, to the best of our knowledge.

\section{Materials and methods}

We implanted carbon ions using the microbeam line of the Center for Micro Analysis of Materials (CMAM) at the Autonomous University of Madrid. The focused ion beam had a size of about $5 \times 3 \mu \mathrm{m}^{2}$ and a beam current $\approx 500 \mathrm{pA}$. The procedure is similar to the one described elsewhere [38]. The target was a high-purity (Electronic Grade) type IIa singlecrystalline diamond (100) plate grown by CVD techniques by Element Six. ${ }^{1}$ Concentrations of nitrogen and boron impurities are reported to be well below $5 \mathrm{ppb}$ and $1 \mathrm{ppb}$, respectively. The diamond crystal was irradiated with a focused ion beam of $9 \mathrm{MeV}^{12} \mathrm{C}^{3+}$ ions with fluences (see Table 1) in the range $4 \times 10^{14}-5.0 \times 10^{16}$ ions $/ \mathrm{cm}^{2}$. We selected a charge state +3 for the ion in order to achieve a magnetic rigidity below the upper limit for the specific configuration of focusing lenses in the microbeam line. Irradiations were conducted along parallel stripes with typical areas of $25 \mu \mathrm{m} \times 500 \mu \mathrm{m}$ (see Fig. 1). The irradiated stripes showed a gray color, the darkening being larger for the highest fluences. The ion beam direction was perpendicular to the unpolished lateral (001) face of the diamond plate along stripes reaching the edges with the top (100) face. Therefore a cross section of the irradiated layers at different depths could be optically accessed from the large polished top face of the sample. According to specifications the surface roughness of the sample was $R_{a}<5 \mathrm{~nm}$ for the polished faces and $R_{a}<200 \mathrm{~nm}$ for the unpolished faces. The simulations did not take into account surface roughness. However, because irradiations were done perpendicular to an unpolished face, the expected effect of the surface roughness will be a broadening of the interfaces by a similar amount $(0.2 \mu \mathrm{m})$. Since this is much smaller than the light wavelength, the spatial resolution of our optical experiments is limited by light diffraction and the effects of surface roughness will not manifest in the results.

Our simulations carried out with the SRIM code [39] show that $9 \mathrm{MeV}$ carbon ions penetrate into diamond to a depth of

\footnotetext{
${ }^{1}$ http://www.e6.com/
} 

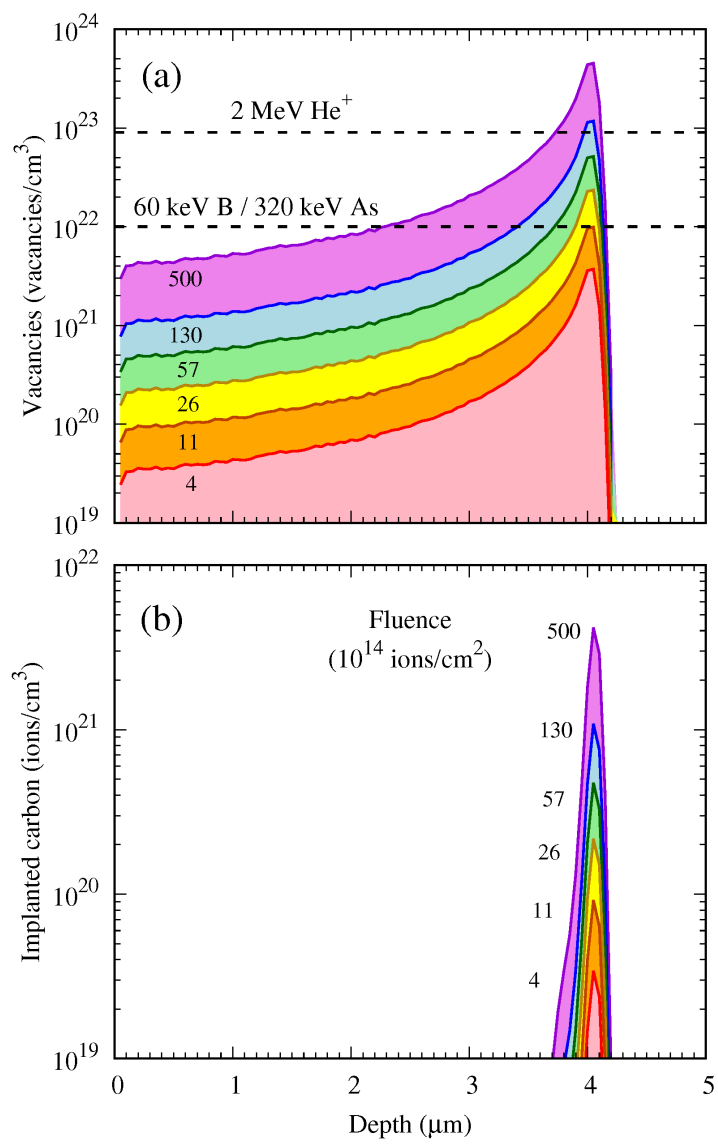

Figure 2: Results of the SRIM simulations for $9 \mathrm{MeV}$ carbon ions implanted on a diamond sample at the indicated values of fluence. (a) Density of vacancies (including ion recoil effects) versus depth. The horizontal dashed lines show the graphitization thresholds reported for $60 \mathrm{keV} \mathrm{B}$ or $320 \mathrm{keV}$ As [23] and $2 \mathrm{MeV}$ $\mathrm{He}^{+}[18]$ ion implantation, respectively. (b) Concentration of implanted carbon ions versus depth. The fluence for each curve is given in units of $10^{14}$ ions $/ \mathrm{cm}^{2}$.

about $4.0 \mu \mathrm{m}$ (Fig. 2). At this depth the calculated density of vacancies and the concentration of implanted carbon present a maximum as a function of depth. The density of vacancies reflects the magnitude of lattice damage. We have used a logarithmic scale for the density of defects because Raman spectroscopy is very sensitive to the lower damage levels.

To heal the damage produced by irradiation, the sample was later annealed in a furnace at $1000^{\circ} \mathrm{C}$ for $1 \mathrm{~h}$. The sample was put in a clean alumina crucible and inserted into a vacuumsealed quartz ampoule, with a low-pressure (100 mbar) of pure helium inside as thermal exchange gas. Diamond has to be annealed in vacuum or an inert gas atmosphere because otherwise, at temperatures above $700^{\circ} \mathrm{C}$, it reacts with oxygen to produce $\mathrm{CO}_{2}$, that escapes as a gas, resulting in a rapid etching of the diamond sample. To avoid possible thermal stresses, heating and cooling ramps, from room temperature to $1000^{\circ} \mathrm{C}$ and return, lasted $3 \mathrm{~h}$ each. After annealing, the darkening of the irradiated stripes diminished but a gray coloration remained, especially for the highest fluences, probably due to the residual lattice defects evidenced by the photoluminescence experiments.

Raman and photoluminescence spectra of the carbon irra-

\begin{tabular}{ccc}
\hline Fluence & $\begin{array}{c}\text { Peak density } \\
\text { of vacancies } \\
\left(10^{21} \mathrm{~cm}^{-3}\right)\end{array}$ & $\begin{array}{c}\text { Peak density } \\
\text { of implanted C } \\
\left(10^{21} \mathrm{~cm}^{-3}\right)\end{array}$ \\
\hline 0 & 0 & 0 \\
4 & 3.65 & 0.034 \\
10 & 8.73 & 0.082 \\
11 & 9.80 & 0.092 \\
21 & 18.7 & 0.17 \\
26 & 23.1 & 0.22 \\
49 & 43.6 & 0.41 \\
57 & 50.8 & 0.47 \\
130 & 116 & 1.08 \\
190 & 169 & 1.58 \\
500 & 445 & 4.17 \\
\hline
\end{tabular}

Table 1: Values of fluence used to implant diamond with 9-MeV carbon ions. The corresponding density of vacancies (including ion recoil effects) and concentration of implanted carbon, obtained from SRIM simulations for the stopping layer (at a depth of $\approx 4.0 \mu \mathrm{m}$ ), are also provided (see Fig. 2).

diated stripes were measured at room temperature before and after the annealing process with a Renishaw Ramascope 2000 spectrometer. The $514.5 \mathrm{~nm}$ excitation wavelength laser line from an argon ion laser was used. Exciting light was focused on the sample surface with a $\mathrm{BH}-2$ Olympus microscope. The objective had a $100 \times$ magnification and a numerical aperture of N.A. $=0.95$. The laser power on the sample surface was of the order of $1 \mathrm{~mW}$. The spectra were recorded in continuous scanning mode because the frequency range was larger than the spectral range of the CCD detector. The integration time for each CCD pixel was 50 and $10 \mathrm{~s}$, respectively, for the Raman and photoluminescence spectra, and each pixel was a point of the spectrum. Each spectrum has several thousand points. Each irradiated stripe was measured at least twice for double checking. Due to the strong dependence of light scattering experiments on surface condition and focus, it is not straightforward to get absolute values of the Raman and photoluminescence intensity. Therefore we have shown the intensity in arbitrary units. Only the intensity values of the same run or mapping can be compared directly.

\section{Results and discussion}

\subsection{Raman spectra}

The Raman spectra measured at different layer depths are represented as three-dimensional surface plots in Fig. 3, with a waterfall graph of the Raman spectra versus layer depth $z$. Fluence values of $2.6 \times 10^{15}, 1.3 \times 10^{16}$ and $5.0 \times 10^{16}$ ions $/ \mathrm{cm}^{2}$, which represent three distinctive regimes, are shown. The front edge corresponds to the sample surface $(z=0)$ and the back edge to unaffected diamond layers $(z=7 \mu \mathrm{m})$ beyond the stopping layer $(z=4 \mu \mathrm{m})$. The structure with a shark dorsal fin appearance is the optical one-phonon Raman peak of crystalline diamond at $1332 \mathrm{~cm}^{-1}$, which is very strong in the unaffected layers but whose intensity decreases significantly for the front irradiated layers because of the disordering of the crystal lattice. After annealing its intensity recovers, specially for the highest 
(a)

(b)
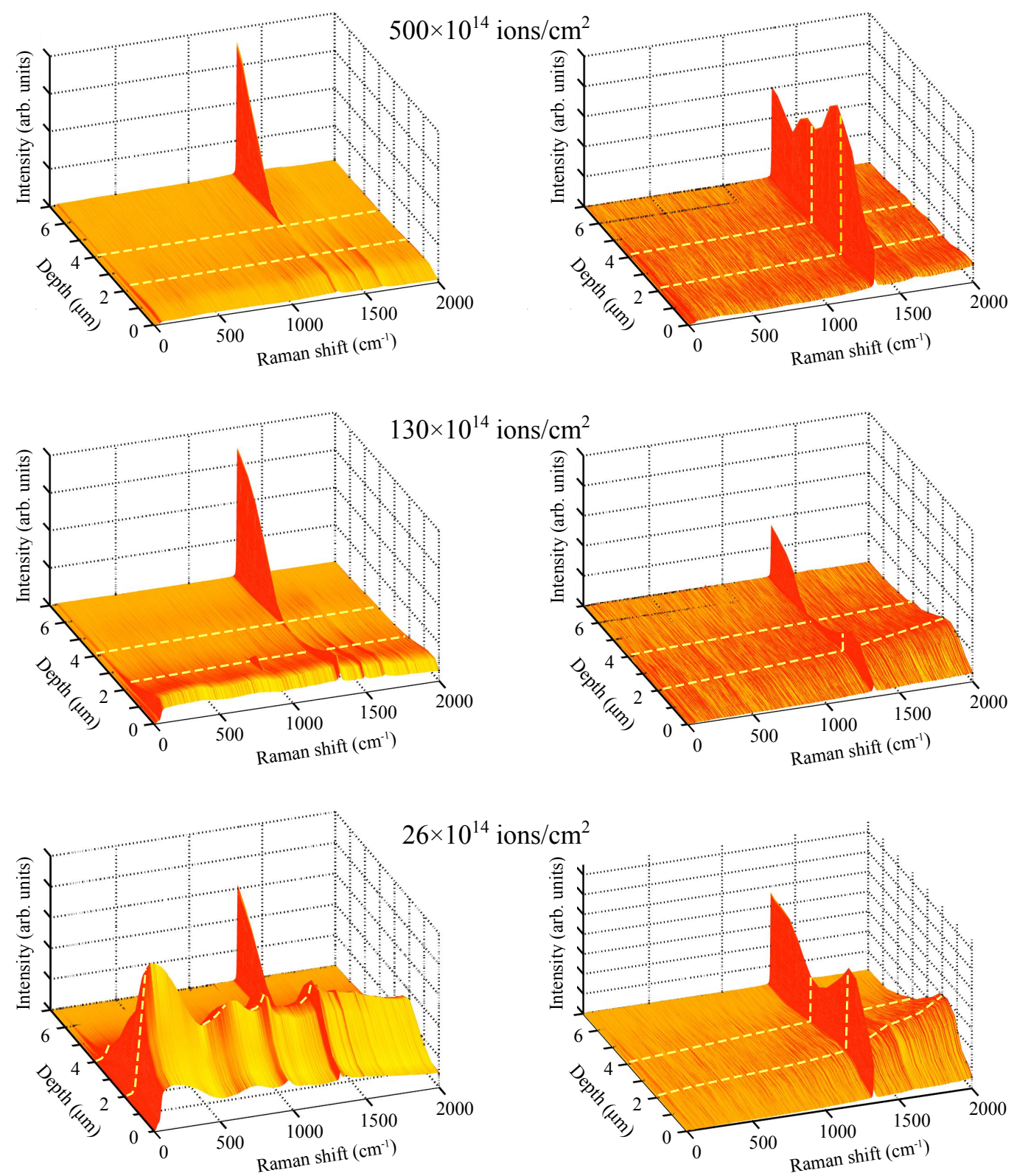

Figure 3: 3D surface plots of the Raman intensity versus Raman shift and layer depth at the indicated fluences before (a) and after (b) annealing. The dashed lines mark the depths of the middle (depth $z=2 \mu \mathrm{m}$ ) and the stopping (depth $z=4 \mu \mathrm{m}$ ) layers. 
fluence, as we will see in more detail later, indicating a healing of the lattice damage. A structured background becomes very intense at low fluences upon irradiation and dies off after annealing.

Raman spectra at the midpoint $(z=2 \mu \mathrm{m})$ of the total ion trajectories, which we will name middle layer from now on, are shown in Fig. 4 for selected fluences in stacked plots. The above-mentioned background becomes stronger at fluences around $2.6 \times 10^{15}$ ions $/ \mathrm{cm}^{2}$ before annealing (Fig. 4(a)). Broad bands are visible at $191,733,973,1096$, and $1367 \mathrm{~cm}^{-1}$. The background weakens for higher damage levels. These bands are due to the photoluminescence emission of the $3 \mathrm{H}$ center $[2,40]$, which consists of self-interstitial carbon atoms produced by irradiation. The background disappears after annealing (Fig. 4(b)) with the healing of the lattice and the diffusion of the interstitial carbon atoms.

At high fluences two small peaks surge above the onephonon peak in the irradiated layers (Fig. 4(a)). The first peak at $1494 \mathrm{~cm}^{-1}$ is due to the formation of a vacancy or a divacancy surrounded by conjugated single and double carboncarbon bonds (the R4/W6 center) [27]. The second peak at $1627 \mathrm{~cm}^{-1}$ is due to the dumbbell defect or $\langle 100\rangle$ split interstitial defect, which consists of an isolated double bonded $\left(s p^{2}\right)$ carbon pair in a carbon site [27]. We have observed the same peaks for the near surface layers in 9-MeV-boron irradiated diamond [37]. These peaks disappear after annealing, indicating that the diffusion of atoms removes most vacancies and interstitial defects produced upon irradiation. For fluences of $5 \times 10^{16}$ ions $/ \mathrm{cm}^{2}$ the one-phonon peak has almost disappeared due to amorphization of the lattice. However, after annealing at $1000^{\circ} \mathrm{C}$ for $1 \mathrm{~h}$ (Fig. 4(b)) the Raman spectrum shows a recovery of the diamond lattice. The one-phonon peak becomes stronger again and the structured background weakens. The recovery is substantial for all the explored fluences. The background on the high energy side is due to photoluminescence emission and will be discussed below. At about $1580 \mathrm{~cm}^{-1}$ there is a very weak and broad band that could be due to the $G$ band of graphite phases, residual interstitial carbon defects or a combination of the two. In any case the amount of graphitic phases would be small and shows that graphitization after annealing has been largely avoided. According to the results published in the literature [23-25], implanted layers will transform to graphite upon annealing beyond a damage threshold of $10^{22}$ vacancies $/ \mathrm{cm}^{3}$ when they are on the surface, that is, when implantation ions have energies of up to tens of $\mathrm{keV}$, because graphite is the most stable phase of carbon at ambient temperature and pressure. However, when the ions have energies in the range of $\mathrm{MeV}$, implanted layers are buried deep inside the diamond matrix. Then graphitization is prevented by the pressure exerted by the diamond matrix when the amorphized layer tries to transform into graphite, because of the much smaller mass density of the latter [18, 26-28]. In fact the weak band at $1580 \mathrm{~cm}^{-1}$ could originate from the graphitization after annealing of the exposed surface of the irradiated stripes on the face used to measure the spectra.

The situation is somehow different for the stopping layer at the end of the ion range $(4.0 \mu \mathrm{m})$ as shown in Fig. 5(a). Here the damage is much heavier (see also Fig. 2) and the Raman spectra are much weaker. Moreover the one-phonon peak disappears already at fluences above $5.7 \times 10^{15}$ ions $/ \mathrm{cm}^{2}$. The structured background is less intense than for the middle layer. After annealing the one-phonon peak recovers its intensity for fluences up to $5.7 \times 10^{15}$ ions $/ \mathrm{cm}^{2}$, indicating an important healing of the crystal structure. However, for fluences around $1.3 \times$ $10^{16}$ ions $/ \mathrm{cm}^{2}$ annealing does not recover the lattice damage, as attested by the weakness of the one-phonon peak (Fig. 5(b)). Surprisingly, for higher fluences around $5 \times 10^{16}$ ions $/ \mathrm{cm}^{2}$ (i.e., a damage about $4.4 \times 10^{23}$ vacancies $/ \mathrm{cm}^{3}$ ), the diamond peak reappears again, indicating that healing is possible beyond the reported critical damage of $9 \times 10^{21}$ vacancies $/ \mathrm{cm}^{3}$. This result indicates that the diamond lattice in the buried layer can recrystallize better when it has been fully disordered. One plausible explanation is that after full amorphization of the lattice the stopping layer recrystallizes following the template provided by the unaffected diamond layers underneath. As stated in the introduction, this phenomenon is called solid phase epitaxy (SPE) and is used frequently in implanted silicon for healing the damaged layers after implantation [14], but has never been clearly observed before in diamond.

To quantify the damage of the lattice we have fitted the optical one-phonon Raman peak to a Lorentzian function. Fig. 6 shows the intensity, position and full width at half maximum (FWHM) as a function of depth and fluence, obtained by a fitting of the 3D surface plots. When the Raman intensity is small or the FWHM is large there can be important errors, but the trend is clear. Before annealing the Raman intensity decreases with fluence for the irradiated layers. For a given fluence it shows a clear dip near the stopping layer reflecting the increasing disorder of the lattice. The increasing disorder is also evidenced by the widening of the peak with fluence at the irradiated layers and, specially, at the stopping layer. Note, however, that the broadening is still very important for the near-surface and intermediate layers, reflecting also a significant damage for these layers [37]. It also shows that Raman spectroscopy is very sensitive to lattice damage of $10^{21}$ vacancies $/ \mathrm{cm}^{3}$. The position of the peak is reduced upon irradiation, reaching a minimum for the stopping layer. This correlates with the increase in the width, following the relationship between width and position due to the anharmonic decay of the optical one-phonon into two acoustic phonons [27, 37, 41], induced by the increasing disorder of the diamond lattice upon irradiation. After annealing there is an important recovery of the lattice except for fluences around $130 \times 10^{14}$ ions $/ \mathrm{cm}^{2}$, for which no recovery is possible. Again, it is clear that the recovery is much better for a high enough fluence, after full amorphization. In fact, the recovery at high fluence is better for the stopping layer than for the middle layer, where the damage is smaller, showing that this enhanced recovery (and maybe SPE) is possible only when the amorphization is complete.

For the surface layer $(z=0 \mu \mathrm{m})$ the position of the onephonon peak presents also a minimum Fig. 6(a), but this is not accompanied by a maximum in the peak width as expected for lattice disorder [27]. Therefore the additional shift must be attributed to some swelling of the lattice caused by the ion irra- 


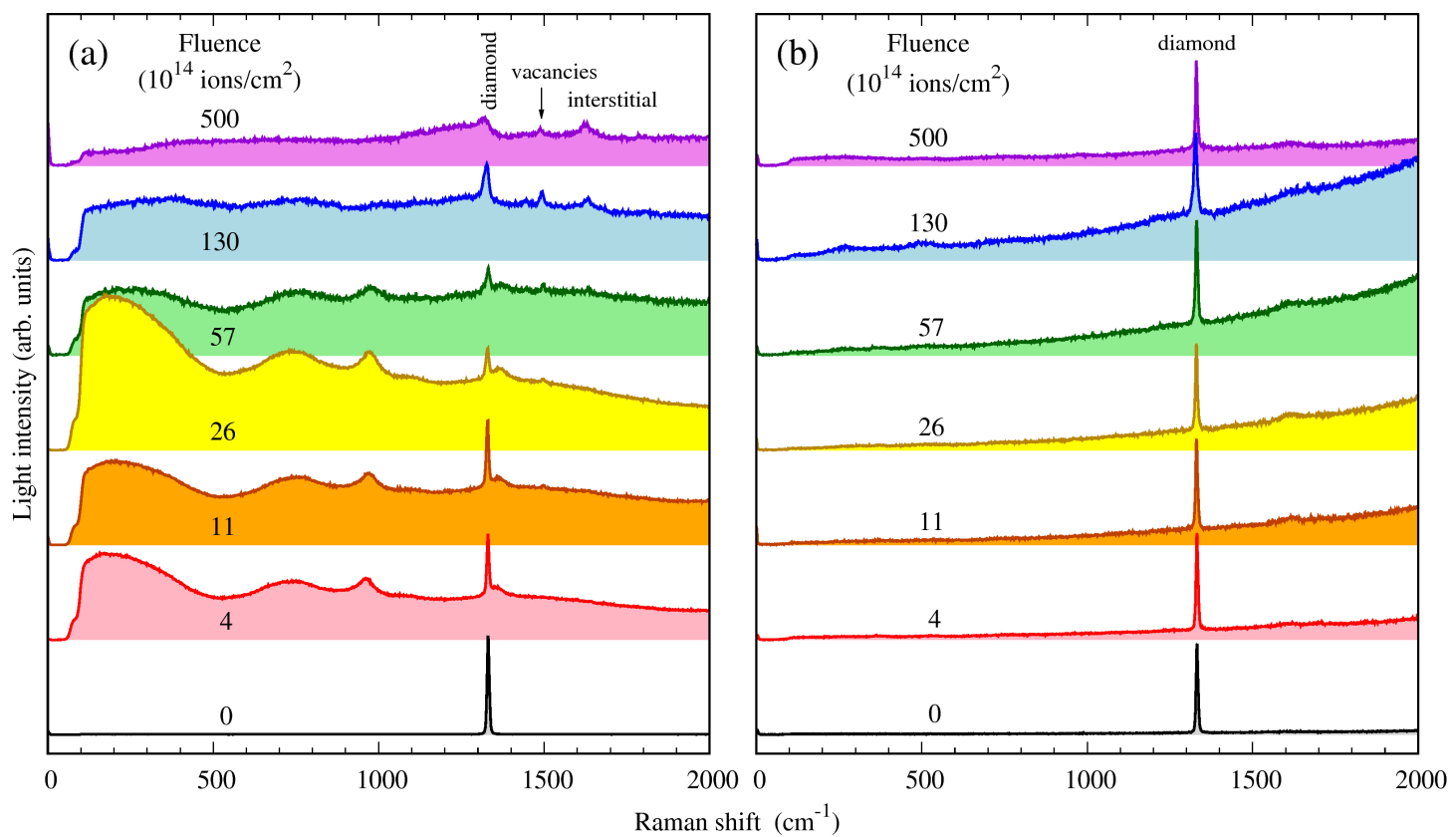

Figure 4: Raman spectra at the middle layer (layer at a depth $z=2 \mu \mathrm{m}$, halfway point of the depth reached by the implanted ions) for the indicated fluences (a) before and (b) after annealing. Text labels in the figure above the spectra indicate the one-phonon peak of diamond $\left(1332 \mathrm{~cm}^{-1}\right)$, the vacancy peak $\left(1492 \mathrm{~cm}^{-1}\right)$ and the interstitial defect $\left(1632 \mathrm{~cm}^{-1}\right)$

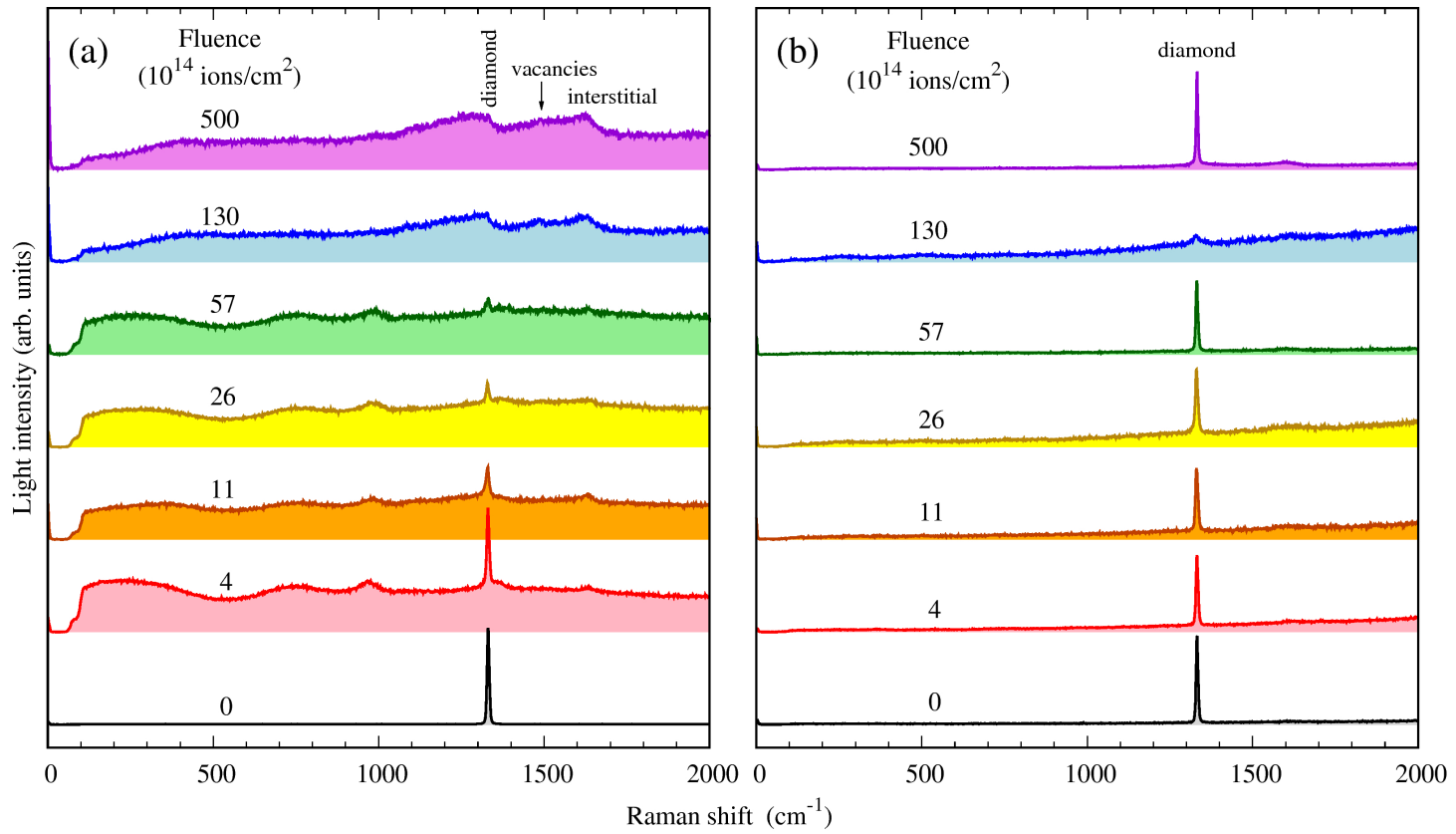

Figure 5: Raman spectra at the stopping layer (at a depth of $z=4 \mu \mathrm{m}$ ) for the indicated fluences (a) before and (b) after annealing. 


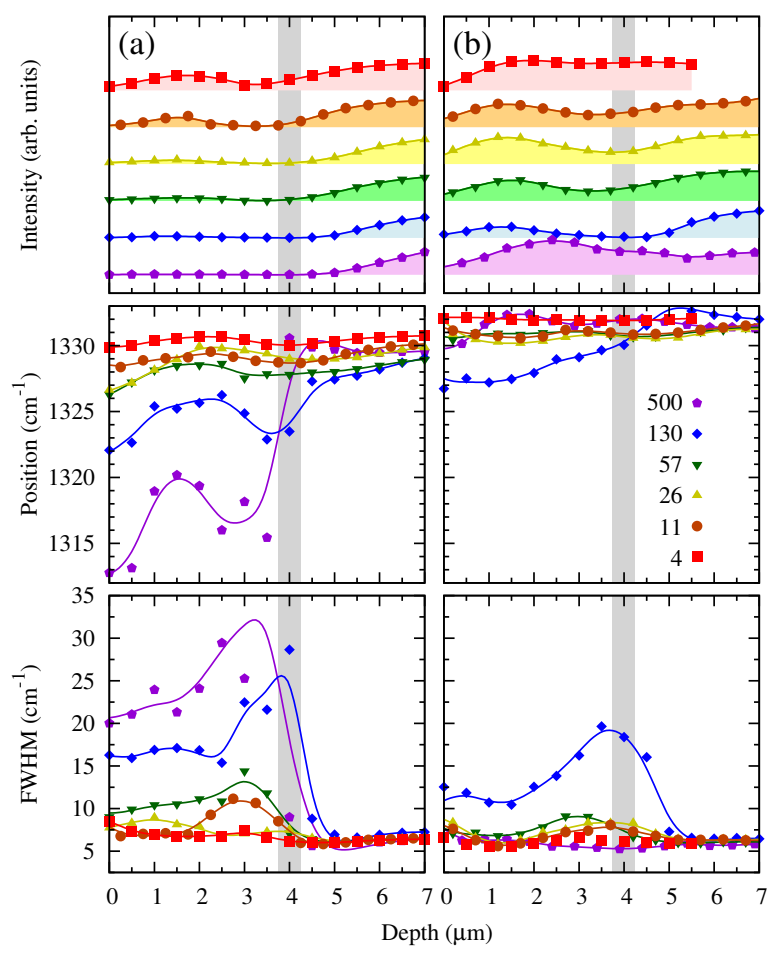

Figure 6: Parameters of a nonlinear least squares fit of the one-phonon diamond Raman band to a Lorentzian function versus layer depth at different fluences before (a) and after (b) annealing. The continuous lines are a guide to the eye. The intensity curves have been vertically offset for clarity and the zero level is marked by the lower edge of the corresponding filled area. The gray vertical band marks the depth of the ion stopping layer according to the SRIM simulations (see Fig. 2). Symbol labels give the corresponding irradiation fluence in units of $10^{14}$ ions $/ \mathrm{cm}^{2}$.

diation. For a fluence of $5 \times 10^{16}$ ions $/ \mathrm{cm}^{2}$ the observed downward shift is about $7 \mathrm{~cm}^{-1}$ with respect to the middle layer. To get a rough estimation of the lattice expansion we can use the relationship with frequency obtained from high pressure experiments reported in the literature. For simplicity we take hydrostatic pressure results. In diamond an equivalent shift of $7 \mathrm{~cm}^{-1}$ is produced by a decrease of $2.5 \mathrm{GPa}$ in the hydrostatic pressure [42] that, taking into account the bulk modulus of diamond [43] $B=444.8 \mathrm{GPa}$, corresponds to a lattice expansion of $0.6 \%$ in volume. Therefore, in our case we infer a similar lattice expansion of $0.6 \%$ in the surface layer for the highest fluence. Volume expansion after ion implantation has been reported before and explained by the amorphization of the material and the formation of vacancies and interstitials [44-46]. Our results indicate that the lattice expansion is more important for the surface layers, where damage and amorphization are smaller. The annealing reduces the swelling, as evidenced by the small difference in the peak position with respect to the middle layer, after the high temperature treatment.

It is also worth studying in detail the variation of the onephonon peak with fluence. In Fig. 7 we have represented the position and the width of the peak versus fluence, obtained from a fit to a Lorentzian lineshape. The fits were done for the middle and the stopping layers, before and after annealing. Before

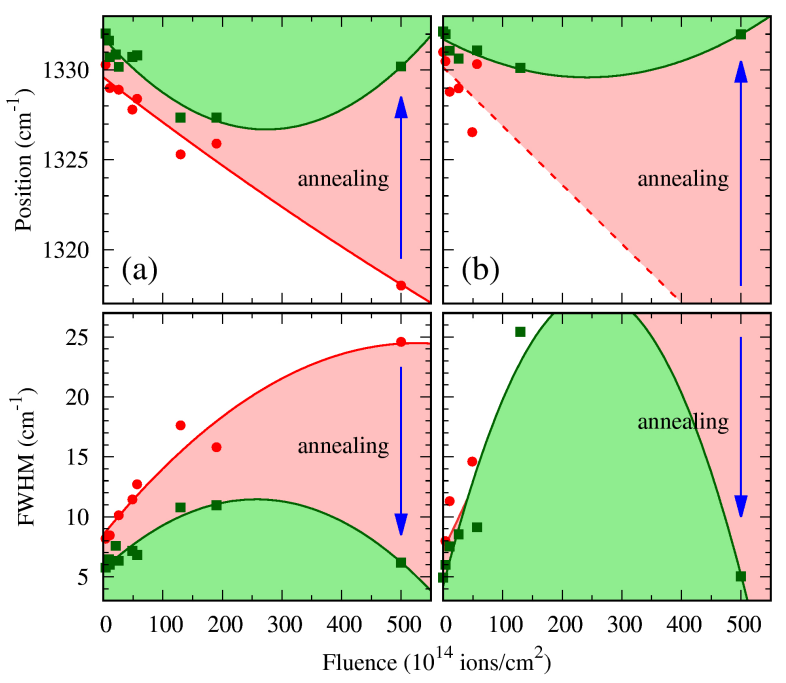

Figure 7: Position and full-width at half-maximum (FWHM) obtained from a fit of the diamond one-phonon Raman peak to a Lorentzian versus the irradiation fluence, before (red full circles) and after (green full squares) annealing. For comparison fit data are shown for the middle (a) and for the stopping (b) layers. The arrows indicate the change with annealing.

annealing the position and the width decrease and increase, respectively, in a monotonic way, indicating an increasing lattice disorder with fluence. After annealing they evolve towards the values of the non-irradiated diamond, indicating a recovery of the lattice. But there is a residual damage left, that increases up to a fluence of about $2 \times 10^{16}$ ions $/ \mathrm{cm}^{2}$. However, for higher fluences the residual damage starts to decrease dramatically, with the peak position and width reaching values of the undamaged diamond crystal for a fluence of $5 \times 10^{16}$ ions $/ \mathrm{cm}^{2}$. For the stopping layer we obtain similar trends, although in this case the damage is larger, the crystalline diamond peak almost disappears and the parameters of the fit for intermediate fluences are less precise. In any case, the healing of the diamond lattice after annealing is better at the highest fluence value than at intermediate fluences. As discussed above we believe that this is due to the onset of solid phase epitaxy. If these results are confirmed for other ions that function as dopants, they would open up the possibility for the use of swift ion irradiation with large fluences to modify the electronic and optical properties of buried layers for diamond device applications.

The excellent work of Orwa et al [27] also studied the damage of diamond by irradiation with energetic ions and its recovery after high temperature annealing by depth-resolved Raman spectroscopy. The main differences with our work is that they irradiated with 3-MeV (and 1-MeV) helium atoms and that they used a single dose value of $10^{17}$ ions $/ \mathrm{cm}^{2}$. On the other hand they studied many annealing temperature values between $300^{\circ} \mathrm{C}$ and $1500^{\circ} \mathrm{C}$. According to their own TRIM simulations they reach a maximum lattice damage of $10^{23}$ vacancies $/ \mathrm{cm}^{3}$. This is interesting because we observe enhanced recrystallization (maybe due to solid phase epitaxy) for a lattice damage above this damage level, at $4.4 \times 10^{23}$ vacancies $/ \mathrm{cm}^{3}$. Following Orwa et al. [27], damage induced by ion irradiation cre- 
ates small and isolated amorphized regions along the ion tracks, together with point defects interspersed between them, in an otherwise largely damage-free crystalline lattice of diamond. Beyond some high level of damage, the amorphized regions overlap and the whole damaged volume amorphizes. Indeed we think that the end-of-range layer must reach a full amorphization for solid phase epitaxy to occur. Otherwise, if pockets of ordered lattice remain, recrystallization leads to a polycrystalline layer. For silicon films, where bonding is weaker, solid phase epitaxy is observed in a limited window of annealing temperatures around $500^{\circ} \mathrm{C}[13,14]$. Therefore in the near future we plan to test other annealing temperatures around $1000^{\circ} \mathrm{C}$ to get the window of temperatures for diamond. It would be also interesting to measure the regrowth rate of the crystalline layers.

\subsection{Photoluminescence spectroscopy}

We have shown before [37] that when a wavelength of $514.5 \mathrm{~nm}(2.41 \mathrm{eV})$ is used for excitation, not far from the Raman bands one can observe also photoluminescence bands. These bands are more sensitive than the Raman peaks to the residual damage after annealing [37]. We have measured the photoluminescence spectra of the irradiated zones in the photon energy range between 1.4 and $2.4 \mathrm{eV}$, as a function of irradiation fluence and layer depth. A typical 3D surface plot of photoluminescence is shown in Fig. 8 before and after annealing. A fluence of $1.1 \times 10^{15}$ ions $/ \mathrm{cm}^{2}$ has been chosen, where the photoluminescence bands are more clear. The front edge of the plot corresponds to the sample surface (depth $z=0$ ). The stopping layer is at a depth of about $4 \mu \mathrm{m}$. The back edge of the plot $(z=7 \mu \mathrm{m})$ corresponds to deeper layers not reached by the ions. They present no significant luminescence emission, which is only visible on the irradiated layers. In these layers the emission is due to the formation of localized defects.

The bands can be more clearly seen in Figs. 9 and 10 that represent the spectra for fluences of $0,4,11,26,57,130$, and $500 \times$ $10^{14}$ ions $/ \mathrm{cm}^{2}$. The bands are the same in the middle (Fig. 9) and in the stopping layers (Fig. 10), in the latter being much weaker, probably because of increased non-radiative recombination. Upon irradiation an intense broad structure appears near $1.6 \mathrm{eV}$, with two narrow bands at $1.671 \mathrm{eV}(741.9 \mathrm{~nm})$ and $1.582 \mathrm{eV}(783.8 \mathrm{~nm})$. The band at $1.671 \mathrm{eV}$ corresponds to the GR1 (general radiation) center, an isolated neutral vacancy $\left(\mathrm{V}^{0}\right)$ [47], that can be produced in diamond by any type of radiation.

After annealing the GR 1 band is replaced by a broad band at $1.60 \mathrm{eV}(775.5 \mathrm{~nm})$. This is the B-band, which is associated with radiation damage [2], indicating that some residual lattice damage remains. The origin of the broad shoulder at $1.93 \mathrm{eV}(642 \mathrm{~nm})$ is not known [30]. The narrow weak bands at $2.026 \mathrm{eV}(612.0 \mathrm{~nm})$ and $2.090 \mathrm{eV}(593.3 \mathrm{~nm})$ correspond to the 594-nm-center reported by Collins et al. [48] and tentatively attributed to interstitial atoms. The sharp peak at $2.245 \mathrm{eV}$, which is stronger for the non-irradiated layers, is the $1332-\mathrm{cm}^{-1}$ onephonon Raman peak of diamond, which has been discussed before. The photoluminescence data indicate that after annealing at $1000^{\circ} \mathrm{C}$ for $1 \mathrm{~h}$ migration of atoms reduce neutral vacancies, but some other residual defects remain. Although the overall intensity decreases for the highest fluences, the photoluminescence spectra, unlike the Raman spectra, do not provide a clear evidence of solid phase epitaxy after annealing. This is a consequence of the photoluminescence being more sensitive to the local structure around the radiative defects and less to the longrange order of the crystal lattice.

\section{Conclusions}

We have studied the lattice damage in a diamond crystal irradiated with 9-MeV carbon ions as a function of fluence and layer depth by Raman and photoluminescence microspectroscopies. In the irradiated layers we observe the formation of neutral vacancies and interstitial defects, and the disordering of the lattice. The damage increases with fluence and reaches a maximum at the stopping layer, which becomes amorphous for fluences well above $10^{16}$ ions $/ \mathrm{cm}^{2}$. After annealing for $1 \mathrm{~h}$ at $1000^{\circ} \mathrm{C}$ the lattice damage partially recovers, leaving some residual damage that increases up to a fluence of about $3 \times 10^{16}$ ions $/ \mathrm{cm}^{2}$. For higher fluences recrystallization of the amorphous layers dramatically reduces the residual damage producing high-quality diamond layers. We have shown that in deeply-buried layers of irradiated diamond, graphitization can be avoided after annealing. Furthermore, when annealed at temperatures around $1000^{\circ} \mathrm{C}$, layers irradiated at fluences of about $5 \times 10^{16}$ ions $/ \mathrm{cm}^{2}$ can recrystallize into high-quality diamond, paving the way for the implantation of diamond at high fluences for diamond device applications in photonics or electronics. One possible interpretation is that recrystallization after annealing at high fluence is taking place by solid phase epitaxy. The latter has been well studied in silicon but has never been observed before in diamond, to the best of our knowledge. We plan to conduct similar experiments with other ions, energies, fluences and annealing temperatures to test this interpretation.

\section{Acknowledgments}

We thank the technical staff of CMAM for operating the tandem ion-beam accelerator. Funding: This work was supported by the Ministerio de Economía y Competitividad (MINECO), Spain [MAT2014-57547-R, MAT2014-54826-C21-R, FIS2014-54498-R, and CTQ-2014-53334-C2-2-R], the María de Maeztu Program for Units of Excellence in R\&D [MDM-2014-0377], and the Comunidad Autónoma de Madrid [NANOFRONTMAG-CM (S2013/MIT-2850)].

\section{References}

[1] P. W. May, Diamond thin films: a 21st-century material, Philos. Trans. R. Soc., A 358 (1766) (2000) 473-495. doi:10.1098/rsta.2000.0542.

[2] A. M. Zaitsev, Optical Properties of Diamond, Springer-Verlag, Berlin, 2001. doi:10.1007/978-3-662-04548-0.

[3] I. Aharonovich, A. D. Greentree, S. Prawer, Diamond photonics, Nat. Photon. 5 (7) (2011) 397-405. doi:10.1038/nphoton.2011.54.

[4] R. Kalish, The search for donors in diamond, Diamond Relat. Mater. 10 (910) (2001) 1749-1755. doi:10.1016/S0925-9635(01)00426-5. 
(a)

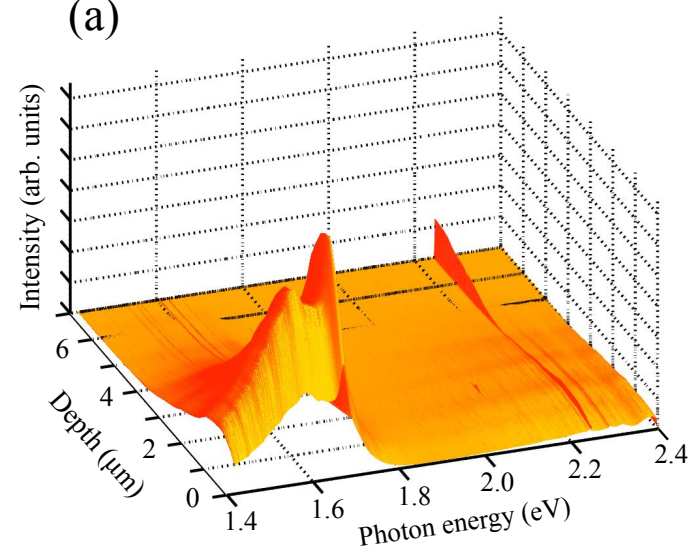

(b)

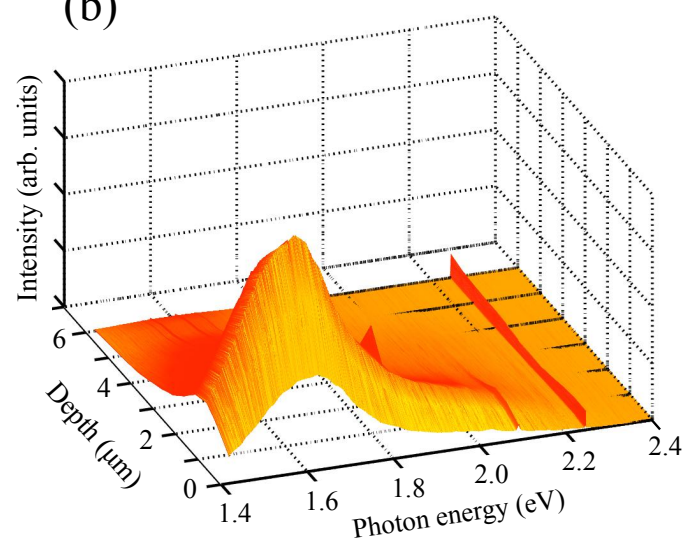

Figure 8: Surface plots of the photoluminescence intensity versus photon energy and layer depth for a fluence of $1.1 \times 10^{15}$ ions $/ \mathrm{cm}^{2}$ before (a) and after (b) annealing.

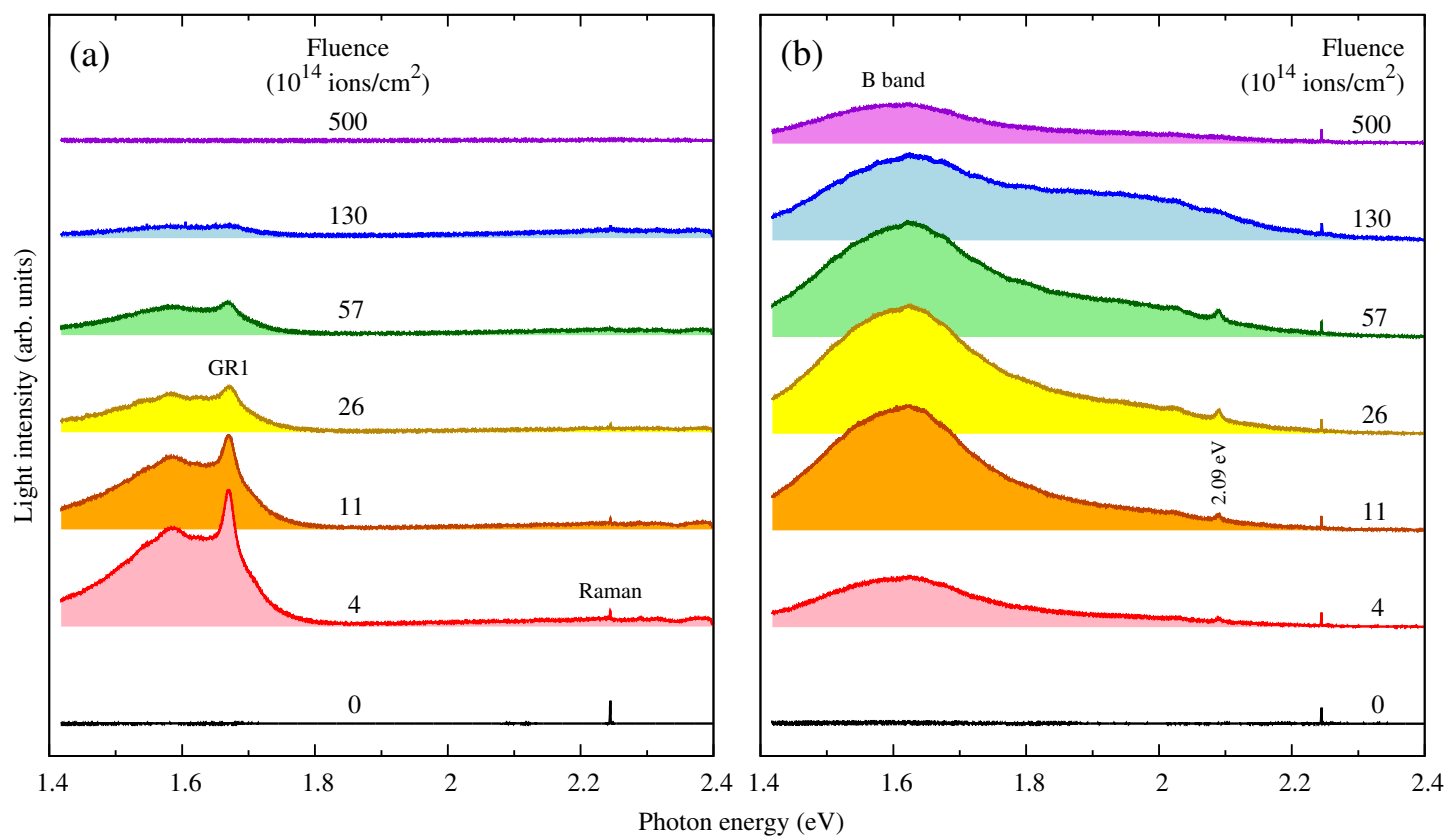

Figure 9: Photoluminescence spectra at the middle layer (at a depth of $z=2 \mu \mathrm{m}$ ) for the indicated fluences (a) before and (b) after annealing. 


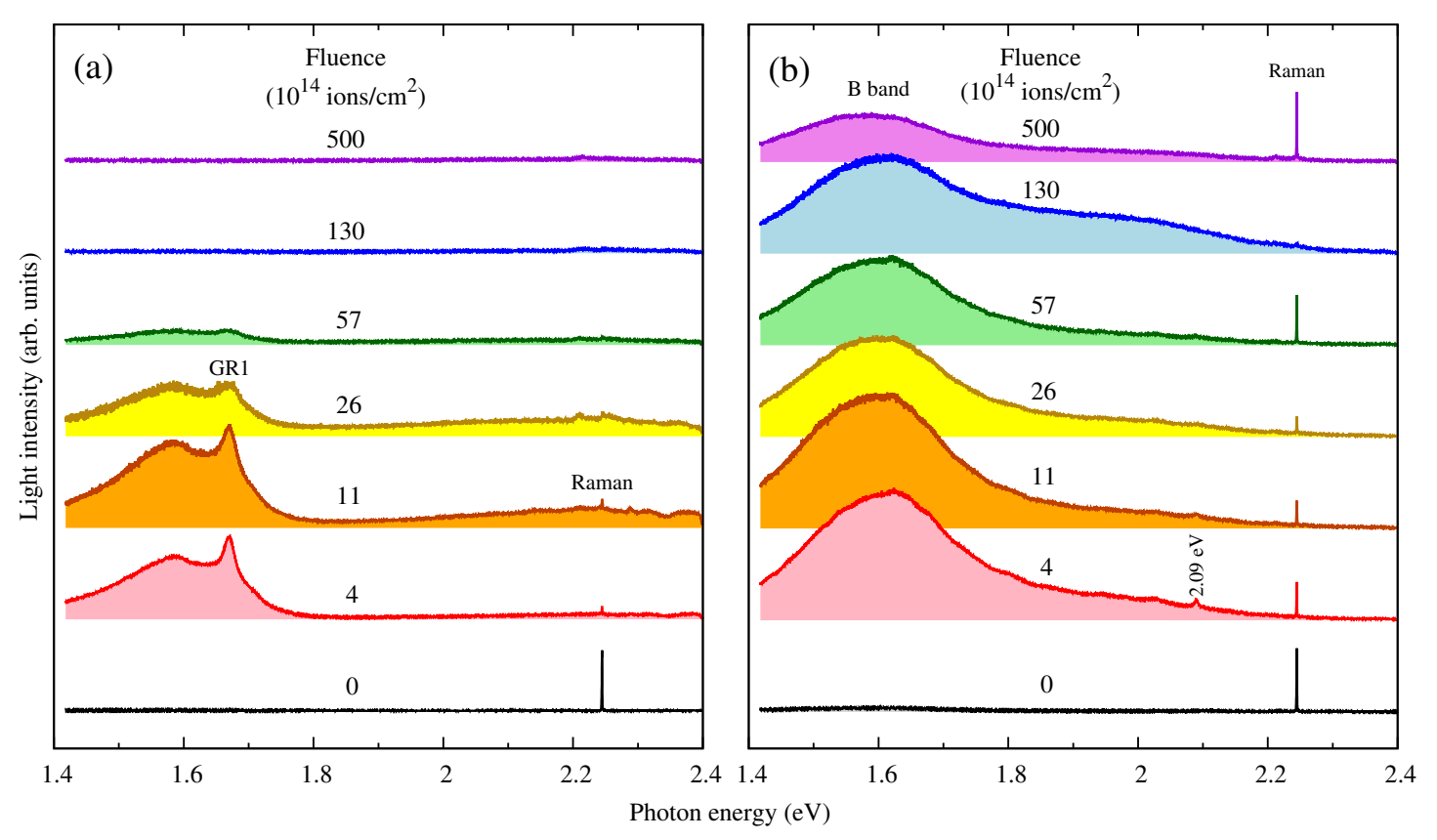

Figure 10: Photoluminescence spectra at the stopping layer (at a depth $z=4 \mu \mathrm{m}$ ) for the indicated fluences (a) before and (b) after annealing.

[5] E. A. Ekimov, V. A. Sidorov, E. D. Bauer, N. N. Mel'nik, N. J. Curro, J. D. Thompson, S. M. Stishov, Superconductivity in diamond, Nature 428 (6982) (2004) 542-545. doi:10.1038/nature02449.

[6] Y. Takano, M. Nagao, I. Sakaguchi, M. Tachiki, T. Hatano, K. Kobayashi, H. Umezawa, H. Kawarada, Superconductivity in diamond thin films well above liquid helium temperature, Appl. Phys. Lett. 85 (14) (2004) 28512853. doi: $10.1063 / 1.1802389$.

[7] M. S. Dresselhaus, R. Kalish, Ion Implantation in Diamond, Graphite and Related Materials, Vol. 22 of Springer Series in Materials Science, Springer, Berlin, 1992. doi:10.1007/978-3-642-77171-2.

[8] J. F. Prins, Using ion implantation to dope diamond an update on selected issues, Diamond Relat. Mater. 10 (9-10) (2001) 1756-1764. doi:10.1016/S0925-9635(01)00427-7.

[9] T. Vogel, J. Meijer, A. Zaitsev, Highly effective $p$-type doping of diamond by MeV-ion implantation of boron, Diamond Relat. Mater. 13 (10) (2004) 1822-1825. doi:10.1016/j.diamond.2004.04.005.

[10] V. Heera, R. Höhne, O. Ignatchik, H. Reuther, P. Esquinazi, Absence of superconductivity in boron-implanted diamond, Diamond Relat. Mater. 17 (3) (2008) 383-389. doi:10.1016/j.diamond.2008.01.057.

[11] A. Lohrmann, S. Pezzagna, I. Dobrinets, P. Spinicelli, V. Jacques, J.F. Roch, J. Meijer, A. M. Zaitsev, Diamond based light-emitting diode for visible single-photon emission at room temperature, Appl. Phys. Lett. 99 (25) (2011) 251106. doi:10.1063/1.3670332.

[12] B. C. Johnson, J. C. McCallum, M. J. Aziz, Solid-phase epitaxy, in: T. F. Kuech (Ed.), Handbook of Crystal Growth, North-Holland, Boston, 2015, Ch. 7, pp. 317-363. doi:10.1016/B978-0-444-63304-0.00007-X.

[13] L. Csepregi, E. F. Kennedy, T. J. Gallagher, J. W. Mayer, T. W. Sigmon, Reordering of amorphous layers of Si implanted with ${ }^{31} \mathrm{P},{ }^{75} \mathrm{As}$, and ${ }^{11} \mathrm{~B}$ ions, J. Appl. Phys. 48 (10) (1977) 4234-4240. doi:10.1063/1.323408.

[14] J.-J. Grob, Ion implantation, in: A. Baudrant (Ed.), Silicon Technologies, John Wiley \& Sons, 2013, Ch. 2, pp. 103-153. doi:10.1002/9781118601044.ch2.

[15] B. A. Fairchild, S. Rubanov, D. W. M. Lau, M. Robinson, I. SuarezMartinez, N. Marks, A. D. Greentree, D. McCulloch, S. Prawer, Mechanism for the amorphisation of diamond, Adv. Mater. 24 (15) (2012) 2024 2029. doi:10.1002/adma.201104511.

[16] G. García, M. Díaz-Híjar, V. Tormo-Márquez, I. Preda, O. PeñaRodríguez, J. Olivares, Structural damage on single-crystal diamond by swift heavy ion irradiation, Diamond Relat. Mater. 58 (2015) 226-229. doi:10.1016/j.diamond.2015.08.014.
[17] S. Lagomarsino, P. Olivero, S. Calusi, D. G. Monticone, L. Giuntini, M. Massi, S. Sciortino, A. Sytchkova, A. Sordini, M. Vannoni, Complex refractive index variation in proton-damaged diamond, Opt. Express 20 (17) (2012) 19382-19394. doi:10.1364/OE.20.019382.

[18] P. Olivero, S. Rubanov, P. Reichart, B. C. Gibson, S. T. Huntington, J. R. Rabeau, A. D. Greentree, J. Salzman, D. Moore, D. N. Jamieson, S. Prawer, Characterization of three-dimensional microstructures in single-crystal diamond, Diamond Relat. Mater. 15 (10) (2006) 1614-1621. doi:10.1016/j.diamond.2006.01.018.

[19] F. Picollo, D. G. Monticone, P. Olivero, B. A. Fairchild, S. Rubanov, S. Prawer, E. Vittone, Fabrication and electrical characterization of threedimensional graphitic microchannels in single crystal diamond, New J. Phys. 14 (5) (2012) 053011. doi:10.1088/1367-2630/14/5/053011.

[20] J. Barzola-Quiquia, T. Lühmann, R. Wunderlich, M. Stiller, M. Zoraghi, J. Meijer, P. Esquinazi, J. Böttner, I. Estrela-Lopis, Fabrication and electrical transport properties of embedded graphite microwires in a diamond matrix, J. Phys. D 50 (14) (2017) 145301. doi:10.1088/13616463/aa6013.

[21] P. Olivero, G. Amato, F. Bellotti, O. Budnyk, E. Colombo, M. Jakšić, C. Manfredotti, v. Pastuović, F. Picollo, N. Skukan, M. Vannoni, E. Vittone, Direct fabrication of three-dimensional buried conductive channels in single crystal diamond with ion microbeam induced graphitization, Diamond Relat. Mater. 18 (58) (2009) 870-876. doi:10.1016/j.diamond.2008.10.068.

[22] J. Forneris, P. Traina, D. G. Monticone, G. Amato, L. Boarino, G. Brida, I. P. Degiovanni, E. Enrico, E. Moreva, V. Grilj, N. Skukan, M. Jakšić, M. Genovese, P. Olivero, Electrical stimulation of non-classical photon emission from diamond color centers by means of sub-superficial graphitic electrodes, Sci. Rep. 5 (2015) 15901. doi:10.1038/srep15901.

[23] C. Uzan-Saguy, C. Cytermann, R. Brener, V. Richter, M. Shaanan, R. Kalish, Damage threshold for ion-beam induced graphitization of diamond, Appl. Phys. Lett. 67 (9) (1995) 1194-1196. doi:10.1063/1.115004.

[24] R. Kalish, A. Reznik, S. Prawer, D. Saada, J. Adler, Ionimplantation-induced defects in diamond and their annealing: Experiment and simulation, Phys. Status Solidi A 174 (1) (1999) 83-99. doi:10.1002/(SICI)1521-396X(199907)174:1;83::AIDPSSA83;3.0.CO;2-3.

[25] R. Kalish, A. Reznik, K. W. Nugent, S. Prawer, The nature of damage in ion-implanted and annealed diamond, Nucl. Instrum. Methods Phys. Res., Sect. B 148 (14) (1999) 626-633. doi:10.1016/S0168-583X(98)00857-X. 
[26] C. Uzan-Saguy, R. Kalish, R. Walker, D. N. Jamieson, S. Prawer, Formation of delta-doped, buried conducting layers in diamond, by highenergy, B-ion implantation, Diamond Relat. Mater. 7 (10) (1998) 14291432. doi:10.1016/S0925-9635(98)00231-3.

[27] J. O. Orwa, K. W. Nugent, D. N. Jamieson, S. Prawer, Raman investigation of damage caused by deep ion implantation in diamond, Phys. Rev. B 62 (9) (2000) 5461-5472. doi:10.1103/PhysRevB.62.5461.

[28] L. H. Willems van Beveren, R. Liu, H. Bowers, K. Ganesan, B. C. Johnson, J. C. McCallum, S. Prawer, Optical and electronic properties of sub-surface conducting layers in diamond created by $\mathrm{MeV}$ Bimplantation at elevated temperatures, J. Appl. Phys. 119 (22) (2016) 223902. doi:10.1063/1.4953583.

[29] J. D. Hunn, S. P. Withrow, C. W. White, D. M. Hembree, Raman scattering from MeV-ion implanted diamond, Phys. Rev. B 52 (11) (1995) 8106-8111. doi:10.1103/PhysRevB.52.8106.

[30] N. Tsubouchi, S.-i. Shikata, Structure, radiation damage and annealing effects in diamond implanted with a high fluence of a few $\mathrm{MeV}$ carbons, Nucl. Instrum. Methods Phys. Res., Sect. B 315 (2013) 165-168. doi:10.1016/j.nimb.2013.04.021.

[31] Y. Mokuno, A. Chayahara, N. Tsubouchi, H. Yamada, S. Shikata, Atomic force microscopy observations of a single crystal diamond surface lifted-off via ion implantation, Diamond Relat. Mater. 31 (2013) 6-9. doi:10.1016/j.diamond.2012.09.001

[32] D. N. Jamieson, S. Prawer, K. W. Nugent, S. P. Dooley, Crosssectional Raman microscopy of MeV implanted diamond, Nucl. Instrum. Methods Phys. Res. B 106 (1-4) (1995) 641-645. doi:10.1016/0168583X(96)80036-X.

[33] S. Prawer, K. W. Nugent, D. N. Jamieson, The Raman spectrum of amorphous diamond, Diam. Relat. Mater. 7 (1) (1998) 106-110. doi:10.1016/S0925-9635(97)00194-5.

[34] L. Nasdala, D. Grambole, M. Wildner, A. M. Gigler, T. Hainschwang, A. M. Zaitsev, J. W. Harris, J. Milledge, D. J. Schulze, W. Hofmeister, W. A. Balmer, Radio-colouration of diamond: a spectroscopic study, Contributions to Mineralogy and Petrology 165 (5) (2012) 843-861. doi:10.1007/s00410-012-0838-1.

[35] O. N. Poklonskaya, S. A. Vyrko, A. A. Khomich, A. A. Averin, A. V. Khomich, R. A. Khmelnitsky, N. A. Poklonskia, Raman scattering in natural diamond crystals implanted with high-energy ions and irradiated with fast neutrons, J. Appl. Spectrosc. 81 (6) (2015) 969-977. doi:10.1007/s 10812-015-0037-8.

[36] A. Deslandes, M. C. Guenette, K. Belay, R. G. Elliman, I. Karatchevtseva, L. Thomsen, D. P. Riley, G. R. Lumpkin, Diamond structure recovery during ion irradiation at elevated temperatures, Nucl. Instrum. Methods Phys. Res., Sect. B 365, Part A (2015) 331-335. doi:10.1016/j.nimb.2015.07.058

[37] F. Agulló-Rueda, M. D. Ynsa, N. Gordillo, A. Maira, D. Moreno-Cerrada, M. A. Ramos, Micro-Raman spectroscopy of near-surface damage in diamond irradiated with 9-MeV boron ions, Diamond Relat. Mater. 72 (2017) 94-98. doi:10.1016/j.diamond.2017.01.010.

[38] M. D. Ynsa, M. A. Ramos, N. Skukan, V. Torres-Costa, M. Jakšić, Highly-focused boron implantation in diamond and imaging using the nuclear reaction ${ }^{11} \mathrm{~B}(\mathrm{p}, \alpha){ }^{8} \mathrm{Be}$, Nucl. Instrum. Methods Phys. Res., Sect. B 348 (2015) 174-177. doi:10.1016/j.nimb.2014.11.036.

[39] J. F. Ziegler, J. P. Biersack, U. Littmark (Eds.), The Stopping and Ranges of Ions in Solids, Pergamon Press, New York, 1985. URL http://www.srim.org/

[40] J. W. Steeds, T. J. Davis, S. J. Charles, J. M. Hayes, J. E. Butler, 3H luminescence in electron-irradiated diamond samples and its relationship to self-interstitials, Diamond Relat. Mater. 8 (10) (1999) 1847-1852. doi:10.1016/S0925-9635(99)00144-2.

[41] M. S. Liu, L. A. Bursill, S. Prawer, R. Beserman, Temperature dependence of the first-order Raman phonon line of diamond, Phys. Rev. B 61 (5) (2000) 3391-3395. doi:10.1103/PhysRevB.61.3391.

[42] S. Prawer, R. J. Nemanich, Raman spectroscopy of diamond and doped diamond, Philos. Trans. R. Soc. London, Ser. A 362 (1824) (2004) 25372565. doi:10.1098/rsta.2004.1451.

[43] E. S. Zouboulis, M. Grimsditch, A. K. Ramdas, S. Rodriguez, Temperature dependence of the elastic moduli of diamond: A Brillouin-scattering study, Phys. Rev. B 57 (5) (1998) 2889-2896. doi:10.1103/PhysRevB.57.2889.

[44] E. W. Maby, C. W. Magee, J. H. Morewood, Volume expansion of ionimplanted diamond, Appl. Phys. Lett. 39 (2) (1981) 157-158. doi:10.1063/1.92645.

[45] J. F. Prins, Ion-implanted structures and doped layers in diamond, Mater. Sci. Rep. 7 (7) (1992) 275-364. doi:10.1016/0920-2307(92)90001-H.

[46] R. A. Khmelnitsky, V. A. Dravin, A. A. Tal, M. I. Latushko, A. A. Khomich, A. V. Khomich, A. S. Trushin, A. A. Alekseev, S. A Terentiev, Mechanical stresses and amorphization of ion-implanted diamond, Nucl. Instrum. Methods Phys. Res. B 304 (2013) 5-10. doi:10.1016/j.nimb.2013.03.030.

[47] A. T. Collins, Intrinsic and extrinsic absorption and luminescence in diamond, Phys. B 185 (1-4) (1993) 284-296. doi:10.1016/09214526(93)90250-A.

[48] A. T. Collins, A. Connor, C.-H. Ly, A. Shareef, P. M. Spear, Hightemperature annealing of optical centers in type-I diamond, J. Appl. Phys. 97 (8) (2005) 083517. doi:10.1063/1.1866501. 